\title{
ON IMBEDDING FINITE-DIMENSIONAL METRIC SPACES
}

\author{
BY
}

\section{STEPHEN LEON LIPSCOMB}

ABSTRACT. The classical imbedding theorem in dimension theory gives a nice topological characterization of separable metric spaces of finite covering dimension. The longstanding problem of obtaining an analogous theorem for the nonseparable case is solved.

1. Introduction. Approximately one decade after the beginnings of the dimension theory for metric spaces, G. Nobeling [6] proved the classical imbedding theorem for separable metric spaces (Theorem 1). Until today, nearly one-half century after the classical theorem, the problem of obtaining an analogous result for the nonseparable case has remained unsolved. Theorem 2 is a solution to this problem.

In 1963, J. Nagata [4] invented a general imbedding theorem for finitedimensional metric spaces. However, the statement of Nagata's theorem involves finite-dimensional subspaces of infinite-dimensional spaces. In contrast, the classical theorem, as well as the new theorem presented here, concerns only subspaces of finite-dimensional spaces. Thus, the new theorem shows that it is not necessary to consider infinite-dimensional spaces for the imbedding problem of finite-dimensional metric spaces. (See Nagata's survey paper [3] for quite a good discussion of the problem.)

The analogy of the classical imbedding theorem and Lipscomb's imbedding theorem goes as follows: For each infinite cardinal $r$ a one-dimensional metric space $J(\tau)$ is constructed which contains rational and irrational points. On the surface, the statements of the two theorems show a general resemblance in that $J(r)$ and the unit interval play similar roles. More deeply, the space $J(r)$ is defined by generalizing a well-known construction of the unit interval (identifying adjacent end points in the Cantor space). Also, the definitions of

Presented to the Society, November 15, 1973; received by the editors November 21, 1973 and, in revised form, July 8, 1974 .

AMS (MOS) subject classifications (1970). Primary 54F45, 54C25; Secondary 54F50, 54B15.

Key words and phrases. Covering dimension, imbedding finitedimensional met:ic spaces, Baire's zero-dimensional space, perfect images of zerodimensional spaces, Cantor's space, decompositions of topological spaces.

Copyright $\odot$ 1975, American Mathematical Society 
rational and irrational points, when phrased in topological terms, remain invariant when we pass to the general case.

In the following we let $I^{2 n+1}$ denote the topological product of $\cdot 2 n+1$ homeomorphic copies of the unit interval. Likewise, we let $J(\tau)^{n+1}$ be the product of $n+1$ copies of the space $J(\tau)$.

Theorem 1. A metric space of weight $r=\kappa_{0}$ is of dimension $\leq n$ if, and only if, it can be imbedded in the set of points of $I^{2 n+1}$ which have at most $n$ rational coordinates.

Theorem 2. A metric space of weight $\tau \geq \boldsymbol{N}_{0}$ is of dimension $\leq n$ if, and only if, it can be imbedded in the set of points of $J(\tau)^{n+1}$ which have at most $n$ rational coordinates.

2. Definitions and well-known theorems. All spaces considered are metric and all maps considered are continuous. The dimension is the covering dimension denoted $\operatorname{dim}$ (see [5]). The cardinality of a set $A$ will be denoted $\kappa(A)$. For an open set $W$ we let $B(W)=\mathrm{cl}(W)-W$, where $\mathrm{cl}$ is the closure operator. For an open collection we let

$$
\text { Bdry } \mathbb{B}=\bigcup\{B(W) \mid W \in \mathbb{B}\} \text {. }
$$

If $\mathbb{R}$ is a collection of subsets of $X$ we will let

$$
\bigcup_{\mathrm{cl}}[\mathbb{R}]=\bigcup\{\mathrm{cl}(W) \mid W \in \mathbb{W}\} \text {. }
$$

If $\mathbb{R}$ is a collection of subsets of a metric space $(X, d)$, then mesh $(\mathbb{R})=$ $\sup \{\operatorname{dia}(W) \mid W \in \mathbb{B}\}$, where

$$
\operatorname{dia}(W)=\sup \{d(x, y) \mid x, y \in W\}
$$

A locally finite open collection $\mathfrak{U}$ is a decomposition of $X$ if $\mathfrak{U}$ is a collection of pairwise disjoint open sets such that $\mathrm{cl}[\mathfrak{U}]=\{\mathrm{cl}(U) \mid U \in \mathfrak{U}\}$ is a cover of $X$.

A map $p: Y \rightarrow Z$ is called perfect if it is a continuous closed surjection and each fiber $p^{-1}(z), z \in Z$, is compact.

We will consider Baire's zero-dimensional space $N(A)$, where $A$ is an infinite set. It is important to us that $N(A)$ is topologically the countable product of discrete spaces $A$. Basic open sets in $N(A)$ of the form

$$
\left\{a_{1}\right\} \Pi \ldots \prod\left\{a_{n}\right\} \prod\left\{A_{i} \mid i>n\right\}
$$

where $a_{i} \in A_{i}$, will be denoted by the ordered $n$-tuple $\left\langle a_{1}, \ldots, a_{n}\right\rangle$. 
Theorem 3. Let $f: Y \rightarrow X$ be a continuous onto quotient map and $p: Y$ $\rightarrow Z$ be continuous. Assume that $p f^{-1}$ is single valued (that is, $p$ is con. stant on each fiber $f^{-1}(x)$ ). Then $p f^{-1}: X \rightarrow Z$ is continuous. Also, $p f^{-1}$ is a closed map if and only if $p(U)$ is closed whenever $U$ is a closed /.in. verse set $\left(U=f^{-1} f(U)\right)$.

3. Constructions analogous to the separable case. If we identify adjacent end points in the Cantor space, then we obtain something homeomorphic to the unit interval. By applying this type of topological construction to the space $N(A)$ we obtain the space $J(\tau)$. This is the content of the following definitions.

Let $\alpha, \beta \in N(A)$. Then $\alpha R \beta$ whenever $\alpha=\beta$ or if $\alpha \neq \beta$, then there exists a natural number $j$ such that for all $k<j, \alpha_{k}=\beta_{k}$ and both conditions (i) and (ii) hold: (i) $\alpha_{j}=\beta_{j+s}$ for all $s \geq 1$; (ii) $\beta_{j}=\alpha_{j+s}$ for all $s \geq$ 1. In this case $j$ is unique and is called the tail index of $\alpha$ and $\beta$. It is easy to show that $R$ is an equivalence relation with the property that any $R$-induced equivalence class contains at most two members. Let $J(\tau), \tau=$ $\boldsymbol{N}(A)$, be the set of $R$-equivalence classes with the quotient topology induced by the natural map $p: N(A) \rightarrow J(\tau)$. The rational points of $J(\tau)$ are those equivalence classes which are doubleton sets and the irrational points of $J(\tau)$ are those that are singleton sets.

Proofs of the following two theorems can be found in [1].

Theorem 4. The natural map $p: N(A) \rightarrow J(\tau)$ is perfect.

Theorem 5. The space $J(\tau)$ is a one-dimensional metric space of weight $\tau$.

A proof of the next theorem can be found in [2].

Theorem 6. Let $n \in\{0,1,2, \cdots\}$. A metric space of weight $\tau \geq \boldsymbol{\kappa}_{0}$ is . of dimension $\leq n$ if it can be imbedded in the set of points in $J(r)^{n+1}$ which have at most $n$ rational coordinates.

4. Decompositions of finite-dimensional spaces. The solution to the problem of imbedding an $n$-dimensional metric space in $J(\tau)^{n+1}, n \geq 0$, is based on the construction of decompositions $\mathbb{B}_{i j}, i \geq 1$ and $1 \leq j \leq n+1$, which have the properties stated in Theorem 7. In this theorem we have used the fact that every metric space is perfectly normal, i.e., every closed subset is a countable intersection of open sets. Also, we use the notation lord $\mathbb{B}_{i j^{\bullet}}$. This is explained as follows: Let $\mathbb{E}$ be the collection of locally finite open collections in the space $X$. For each $x \in X$ and each $C \in \mathbb{S}$ 
such that $x$ is in the closure of a member of $C$, we define lord $_{x} C$ as the smallest $k$ in $\{1,2, \ldots\}$ for which it is true that there is an open set $G$ containing $x$ such that $G$ meets at most $k$ members of $C$. We obtain a function lord and a dimension diml in the same way that the function ord and dimension dim are obtained from ord $x^{\cdot}$ (Using a result in Ostrand's paper [7] which says that the definition of dim can be based on locally finite open collections instead of finite open collections, we can show that $\operatorname{dim} X=\operatorname{diml} X$ whenever $X$ is a normal Hausdorff space.)

Theorem 7. Let $n \in\{0,1,2, \ldots\}$. Let $X$ be an $n$-dimensional metric space of infinite weight $\tau=\boldsymbol{X}(A)$. Let $\left\{X_{j} \mid 1 \leq j \leq n+1\right\}$ be a pairwise dis. joint collection of zero-dimensional subsets of $X$ such that $X=\bigcup\left\{X_{j} \mid 1 \leq\right.$ $j \leq n+1\}$. For each closed subset $F$ of $X$ let $\left\{G_{i}(F) \mid i \geq 1\right\}$ be a collection of open sets such that $F=\bigcap\left\{G_{i}(F) \mid i \geq 1\right\}$. Then there exist decompositions $\mathbb{B}_{i j}=\{W(\alpha) \mid \alpha \in A\}, i \geq 1$ and $1 \leq j \leq n+1$, with the following properties: For each $i, i \geq 1$,

$(1)_{i}$ lord $\mathbb{B}_{i j} \leq 2$ for $1 \leq j \leq n+1$;

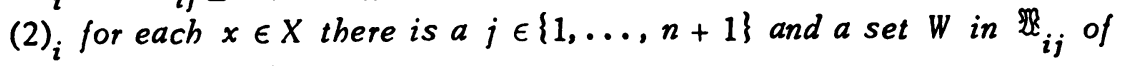
diameter less than $1 / i$ which contains $x$ and is contained in a member of $\mathbb{B}_{k j}$ for each $k<i$;

(3) $_{i}$ if $x \in \mathrm{Bdry} \mathbb{P}_{i j}$, then there exist distinct members $W_{1}, W_{2} \in \mathbb{R}_{i j}$ such that $x \in B\left(W_{1}\right) \cap B\left(W_{2}\right)$;

(4) for each $j, 1 \leq j \leq n+1$, 促 ${ }_{i j}$ covers $X_{j}$;

$(5)_{i}$ for each $k<i$ and $1 \leq j \leq n+1$, Bdry $\mathbb{2}_{i j} \cap \mathrm{Bdry} \mathbb{R}_{k j}=\varnothing$;

$(6)_{i}$ if $B\left(W_{1}\right) \cap B\left(W_{2}\right) \neq \varnothing$ for distinct $W_{1}, W_{2} \in \mathbb{W}_{i j}$, then either $W_{1}$ is a subset of a member of $\mathfrak{K}_{k j}$, for each $k<i$, or $W_{2}$ is a subset of a member of $\mathbb{B}_{k j}$, for each $k<i$;

(7) $)_{i}$ for $k<i$ and $1 \leq j \leq n+1, W \in \mathbb{B}_{i j}$ meets at most two members of $\mathbb{R}_{k j}$, and, for all but at most one of the $k<i$, there exists a member of $\mathbb{B}_{k j}$ which contains $W$;

(8) $)_{i}$ for $k<i$ and $1 \leq j \leq n+1$, if $W_{1}, W_{2} \in \mathbb{R}_{k j}$ are distinct and $B\left(W_{1}\right)$ $\cap B\left(W_{2}\right) \neq \varnothing$, then there is a $W_{3} \in \mathbb{B}_{i j}$ such that

$$
B\left(W_{1}\right) \cap B\left(W_{2}\right) \subset W_{3} \subset G_{i}\left(B\left(W_{1}\right) \cap B\left(W_{2}\right)\right)
$$

(9) $_{i}$ for $k<i, W \in \mathbb{B}_{i j}$ meets Bdry $\mathbb{B}_{k j}$ only if there is a unique distinct pair $W_{1}, W_{2} \in \mathbb{B}_{k j}$ such that $B\left(W_{1}\right) \cap B\left(W_{2}\right) \subset W$.

The proof of this theorem is presented at the end of $\$ 6$. 
5. Imbedding $n$-dimensional metric spaces in $J(\tau)^{n+1}$. In this section we outline the method that we use to imbed an $n$-dimensional metric space $X$ into $J(r)^{n+1}$. The details and definitions are contained in the last section of the paper.

The map $p^{n+1}: N(A)^{n+1} \rightarrow J(\tau)^{n+1}$ is defined to be the product map which has the natural map $p: N(A) \rightarrow J(r)$ as each of its factor maps.

We use the decomposition theorem, Theorem 7 , twice. First, we define a continuous quotient map $h$ from $T \subset N(A)^{n+1}$ onto $X$. Secondly, we define a homeomorphism $\theta$ from $T$ into $N(A)^{n+1}$. The map $\theta: T \rightarrow \theta(T)$ has an important property: For each $x \in X$ there is a $z \in J(\tau)^{n+1}, z$ has at most $n$ rational coordinates, such that $\theta$ maps the set $h^{-1}(x)$ onto the set $\left(p^{n+1}\right)^{-1}(z)$.

For each $x \in X$ we can use this property to determine a $z \in J(r)^{n+1}$. We denote this correspondence $q: X \rightarrow J(\tau)^{n+1}$. Since $\left\{h^{-1}(x) \mid x \in X\right\}$ is a partition of $T$ and $\theta: T \rightarrow \theta(T)$ is one-one with the property above, we can easily see that $q$ is one-one and well defined. Also, since $\theta$ takes each set $h^{-1}(x)$ onto a set $\left(p^{n+1}\right)^{-1}(z)$ we can see that $\theta(T)$ is a $\left(p^{n+1}\right)$-inverse set, i.e., $\left(p^{n+1}\right)^{-1} p^{n+1}(\theta(T))=\theta(T)$.

The situation we now have is best described with the aid of Figure 1. In the figure we see the maps $p^{n+1}, b, \theta$, and $q$ as we have discussed above.

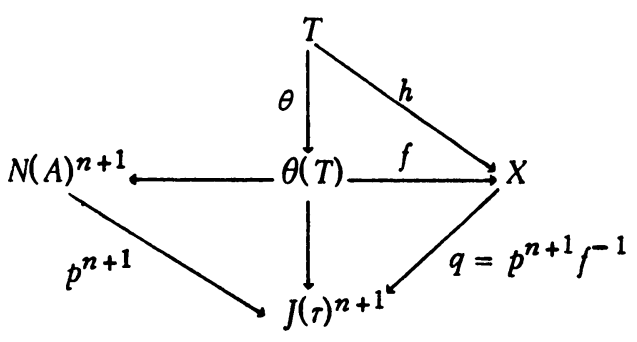

Figure 1

The map $f$ is defined as the composition map $h\left(\theta^{-1}\right)$. Since $\theta$ is a homeomorphism, and, $h$ is continuous and quotient, we see that $f$ is a continuous quotient map. Further, since $p$ is perfect we know that $p^{n+1}$ is perfect. Thus, since $\theta(T)$ is a $\left(p^{n+1}\right)$-inverse set, $p^{n+1}$ restricted to $\theta(T)$ is a closed map. Hence, we can make an easy application of Theorem 3 to show that $q=p^{n+1} f^{-1}$ is a homeomorphism.

6. Lemmas for the decomposition theorem. We will prove Theorem 7 with an induction argument based on the index $i=1,2, \ldots$. However, before 
the inductive step can be set up we must introduce some new concepts and prove a few intermediate results.

Lemma 1. Let $n \in\{-1,0,1, \cdots\}$. Let $Y$ be a closed subspace of $X$ of dimension $\leq n$, and let $Z$ be an open subset of $X$ such that $Y \subset Z$. Let $\mathfrak{u}^{\prime}$ $=\left\{U_{\alpha}^{\prime} \mid \alpha \in \bar{A}\right\}$ be a locally finite open cover of $X$. Then there is an open cover $\mathfrak{U}=\left\{U_{d} a \in A\right\}$ of $X$ which precisely refines $\mathfrak{U}^{\prime}$, and an open set $G, Y$ $\subset G \subset \mathrm{cl}(G) \subset Z$, such that for each $a \in A, U_{\alpha}^{\prime}-U_{a} \subset \mathrm{cl}(G)$. Also, each point of $\mathrm{cl}(G)$ meets $U_{a}$ for at most $n+1$ of the $a \in A$.

Proof. Let $B=\left\{\alpha \in A \mid U_{a}^{\prime} \cap Y \neq \varnothing\right\}$. Then $\left\{U_{a}^{\prime} \mid \alpha \in B\right\}$ is a locally finite open cover of $Y$. Hence, there is an open precise refinement $B=\left\{V_{a} \mid a \in B\right\}$ of $\left\{U_{a}^{\prime} \mid a \in B\right\}$ which covers $Y$ and has the property that ord $\mathbb{B} \leq n+1$. (We can use arguments similar to those given in the appendix of Ostrand's paper

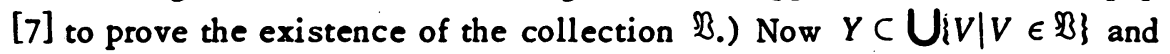
we can choose $G$ open such that

$$
Y \subset G \subset \operatorname{cl}(G) \subset(\bigcup\{V \mid V \in \mathbb{R}\}) \cap Z
$$

Let $U_{a}=\left[U_{\alpha}^{\prime}-\operatorname{cl}(G)\right] \cup V_{\alpha}$ for $\alpha \in B$ and $U_{\alpha}=U_{\alpha}^{\prime}-\operatorname{cl}(G)$ for $\alpha \epsilon A-B$. Then clearly $\mathfrak{U}=\left\{U_{a} \mid a \in A\right\}$ and $G$ satisfy the required conditions.

Let $B=\{V(\alpha) \mid \alpha \in A\}$ be a locally finite open cover of $X$. Let $k \epsilon$ $\{1,2, \ldots\}$. Then $O$ is a $k$-node of $B$ if, and only if, there exist distinct $a_{1}$, $\ldots, \alpha_{k}$ in $A$ such that

$$
O=\left[V\left(\alpha_{1}\right) \cap \ldots \cap V\left(\alpha_{k}\right)\right]=\left[\bigcup\left\{V(\beta) \mid \beta \in A ; \beta \neq \alpha_{r} ; 1 \leq r \leq k\right\}\right] .
$$

We will denote the collection of $k$-nodes of $\mathfrak{B}$ as $\bigcap_{k}(\mathbb{B})$. It is clear that $\mathcal{U}\left\{\bigcap_{k}(\mathbb{B}) \mid k \geq 1\right\}$ is a locally finite collection of pairwise disjoint sets which covers $X$. Also, an important property of these $k$-nodes can be stated as follows: If $x \in \mathrm{cl}(0)-0$, where $0 \in \Omega_{k}(\Re)$, then there is an $m<k$ such that $x \in P \in \bigcap_{m}(\mathbb{B})$. We will call this property the nodal closure property and denote it by N.C.P.

Let $\mathcal{F}=\left\{F_{\beta} \mid \beta \in B\right\}$ be a collection of sets which precisely refine $B=$ $\left\{G_{\beta} \mid \beta \in B\right\}$. In this case we say that $\mathfrak{F}$ shrinks $\mathbb{B}$. Also, a collection $\mathfrak{U}=$ $\left\{U_{\beta} \mid \beta \in B\right\}$ puffs-up $\mathfrak{F}$ in $\&$ whenever $F_{\beta} \subset U_{\beta} \subset G_{\beta}$ for each $\beta \in B$. Further, if $F_{\beta} \subset O_{\beta}$ for each $\beta \in B$, where $\left\{O_{\beta} \mid \beta \in B\right\}$ is a subcollection of $\bigcup\left\{\bigcap_{k}(B) \mid k \geq 1\right\}$ for some locally finite open cover $B$ of $X$, then the canonical $(\mathfrak{f}, \mathfrak{B})$ collection is the open collection $\left\{G_{\beta} \mid \beta \in B\right\}$ where $G_{\beta}=V\left(\alpha_{1}\right) \cap$ $\ldots \cap V\left(a_{k}\right)$ whenever

$$
F_{\beta} \subset O_{\beta}=\left[V\left(a_{1}\right) \cap \ldots \cap V\left(a_{k}\right)\right]-\left[\bigcup\left\{V(\gamma) \mid \gamma \in A ; \gamma \neq a_{r} ; 1 \leq r \leq k\right\}\right] .
$$


It is clear that when $\mathfrak{F}$ shrinks a collection of nodes of $\mathfrak{B}$, then $\mathfrak{F}$ shrinks the canonical $(\mathfrak{F}, \mathfrak{B})$ collection.

Lemma 2. Let $1 \leq j \leq n+1$. Let $\mathfrak{U}$ be a locally finite open cover of $X$ of ord $\leq n+1$. For each $j$ let $X_{j}$ be a zero-dimensional subspace of $X$. For each $j$ let $F_{j}$ be a closed subset of $X$ such that (U\{O|O $\left.\left.\in \bigcap_{j}(\mathfrak{U})\right\}\right)$ does not meet $F_{j}$. Then there are $n+1$ discrete open collections $\mathfrak{U}_{1}(1), \ldots$, $\mathfrak{U}_{n+1}(1)$ such that $\bigcup\left\{\mathfrak{u}_{j}(1) \mid 1 \leq j \leq n+1\right\}$ covers $X$ and refines $\mathfrak{u}$. Also, for each $j$, Ucl $\left[\mathfrak{u}_{j}(1)\right] \cap F_{j}=\varnothing$ and $B(U) \cap X_{j}=\varnothing$ for all $U \in \mathfrak{U}_{j}(1)$. Further, if $\mathfrak{U}=\left\{U_{a} \mid \alpha \in A\right\}$, then for each $j: \mathcal{N}\left(\mathfrak{U}_{j}(1)\right) \leq \boldsymbol{N}(A)\left[\boldsymbol{\alpha}\left(\mathfrak{U}_{j}(1)\right)<\mathcal{N}_{0}\right]$ whenever $A$ is infinite [finite].

Proof. Let $1 \leq k \leq n+1$ and $1 \leq j \leq n+1$. For $j<k$ we suppose that the desired collections $\mathfrak{u}_{j}(1)$ have been defined such that $\mathfrak{B}(k)=\bigcup \mathfrak{U} \mathfrak{U}_{j}(1) \mid$ $j<k\}$ covers all the nodes of $\mathfrak{U}$ of order less than $k$. Now if $Q=X-$ $\bigcup\{U \mid U \in \mathfrak{B}(k)\}$, then it follows from the N.C.P. that $F=\left\{O \cap Q \mid O \in \bigcap_{k}(\mathfrak{U})\right\}$ is a discrete closed collection. This closed collection $F$ shrinks the open canonical $(\mathfrak{F}, \mathfrak{U}$ ) collection. We call this canonical collection $\mathbb{B}$. Then by collectionwise normality of $X$, zero-dimensionality of $X_{k}$, and the fact that $F_{k} \cap\left(\bigcup\left\{O \mid O \in \bigcap_{k}(\mathfrak{U})\right\}\right)=\varnothing$, there is a discrete open collection $\mathfrak{U}_{k}(1)$ which puffs-up $\mathfrak{F}$ in $\&$ such that $U \in \mathfrak{U}_{k}(1)$ implies that $\mathrm{cl}(U) \cap F_{k}=\varnothing$ and $B(U)$ $\cap X_{k}=\varnothing$. Thus $\mathfrak{U}_{k}(1)$ has been constructed using $\mathfrak{U}_{j}(1), j<k$, and therefore the induction step is complete. The properties of $\mathfrak{U}_{1}(1), \ldots, \mathfrak{U}_{n+1}(1)$ clearly follow.

If $n \in\{0,1, \ldots\}$, then $F_{1}, \ldots, F_{n+1}$, being closed subsets of $X$, satisfy the property $P(n-1)$ whenever $\operatorname{dim}\left(F_{j_{1}} \cap \cdots \cap F_{j_{k}}\right) \leq n-k$ for distinct $j_{1}, \ldots, j_{k}$. In addition to the property $P(n-1)$ we will need the definition of separated collection. Let $\Re=\left\{N_{\beta} \mid \beta \in B\right\}$ be a collection of subsets of $X$. Then $\Re$ is a separated collection whenever each pair of sets in $\Re$ is separated, i.e.,

$$
\left[\operatorname{cl}\left(N_{\beta}\right) \cap N_{\gamma}\right] \cup\left[N_{\beta} \cap \operatorname{cl}\left(N_{\gamma}\right)\right]=\varnothing \quad \text { for } \beta, \gamma \in B \text { with } \beta \neq \gamma \text {. }
$$

Since $(X, d)$ is metric we can see that a locally finite separated collection $\Re$ can be puffed-up to a pairwise disjoint open collection. (If

$$
M_{\beta}=\left\{x \in X \mid d\left(x, N_{\beta}\right)<d\left(x, \bigcup\left\{c l\left(N_{\gamma}\right) \mid \gamma \neq \beta ; \gamma \in B\right\}\right)\right\},
$$

then $M=\left\{M_{\beta} \mid \beta \in B\right\}$ is the desired collection.)

Lemma 3. Let $n \in\{0,1, \ldots\}$ and let $F$ be a closed n-dimensional subspace of $X$. Let $F_{1}, \ldots, F_{n+1}$ be closed subsets of $F$ which satisfy 
$P(n-1)$. Let $B^{\prime}$ be a locally finite open cover of $X$ indexed on $A$. Then there is a locally finite open collection $\mathfrak{U}$ refining $\mathfrak{B}^{\prime}$ and covering $X$ such that $\boldsymbol{x}(\mathfrak{U}) \leq \boldsymbol{N}(A)\left[\boldsymbol{N}(\mathfrak{U})<\boldsymbol{\aleph}_{0}\right]$ when $A$ is infinite [finite];

(10) $F \subset$ int (U $\left.\left\{O \mid O \in \bigcirc_{j}(\mathfrak{U}) ; 1 \leq j \leq n+1\right\}\right)$ where int = interior; and

(11) for each $j, 1 \leq j \leq n+1, F_{j} \subset X-\bigcup\left\{0 \mid O \in \bigcap_{j}(\mathfrak{M})\right\}$.

Proof. Let $n=0$. In this case we can apply Lemma 1 with $\mathfrak{U}^{\prime}=\mathfrak{B}^{\prime}, Z$ $=X$, and $Y=F$. Thus the first step in the induction proof is complete. We now suppose that the statement is true for $n-1$, where $n>0$, and proceed to show that it is true for $n$. To begin, we let $H=F_{1} \cup \ldots \cup F_{n+1}$ and for each $j, 1 \leq j \leq n$, we let $H_{j}=\bigcup\left\{F_{k} \cap F_{j} \mid j<k \leq n+1\right\}$. Then $H$ is an $(n-1)$ dimensional closed subspace of $X$. Also, $H_{1}, \ldots, H_{n}$ are closed subsets of $H$ which satisfy $P(n-2)$. Further, $B^{\prime}$ is a locally finite open cover of $X$ indexed on $A$. Therefore, by the inductive hypothesis, there is a locally fi-

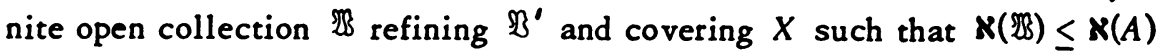
$\left[\boldsymbol{X}(\mathbb{B})<\boldsymbol{\aleph}_{0}\right]$ when $A$ is infinite [finite];

(12) $H \subset$ int $\left(\bigcup\left\{O \mid O \in \bigcap_{j}(\mathbb{B}) ; j \leq n\right\}\right)$; and

(13) for each $j, 1 \leq j \leq n, H_{j} \subset X-\bigcup\left\{0 \mid O \in \bigcap_{j}(\mathbb{R})\right\}$.

Let $\Re_{j}, 1 \leq j \leq n+1$, be the collection $\left\{O \cap F_{j} \mid O \in \bigcap_{j}(\mathbb{B})\right\}$. Then $\Re=$ $\mathcal{U}\left\{\Re_{j} \mid 1 \leq j \leq n+1\right\}$ is a separated collection which is locally finite. (The proof of this fact is given below.) For conciseness of notation we now index $\Re$ as $\left\{N_{\beta} \mid \beta \in B\right\}$. Since $\Re$ is a locally finite separated collection there is a pairwise disjoint open collection $\mathbb{M}=\left\{M_{\beta} \mid \beta \in B\right\}$ such that $\Re$ shrinks $\mathbb{M}$. We let $\left\{G_{\beta} \mid \beta \in B\right\}$ be the canonical $(\Re, \mathbb{B})$ collection. Then $\left\{G_{\beta} \mid \beta \in B\right\}$ is a locally finite open collection and $\Re$ shrinks $\left\{G_{\beta} \mid \beta \in B\right\}$. We define an open collection $\mathcal{Z}=\left\{L_{\beta} \mid \beta \in B\right\}$. Let $\beta \in B$ be fixed and suppose $O \cap F_{j}=N_{\beta} \epsilon$ $\Re_{j}$ for some $j \in\{1, \ldots, n+1\}$. Then we define

$$
\begin{aligned}
L_{\beta}= & {\left[M_{\beta} \cap G_{\beta} \cap \operatorname{int}\left(\bigcup\left\{O \mid O \in \bigcap_{k}(\mathbb{R}) ; k \leq n\right\}\right)\right] } \\
& -\left[\bigcup\left\{F_{k} \mid k>j\right\} \cup \cup\left\{P \mid P \in \bigcap_{k}(\mathbb{B}) ; k<j\right\}\right] .
\end{aligned}
$$

Now $\Omega$ is an open collection and $\Re$ shrinks $?$. (The proof of the fact is given below.) Obviously, we can see that $\kappa(\Omega) \leq \kappa(A)\left[\kappa(\Omega)<\mathcal{K}_{0}\right]$ when $A$ is infinite [finite]. We let $\mathfrak{U}^{\prime}=\mathbb{B} \cup\left\{\right.$. Since $\left\{\right.$ shrinks $\left\{G_{\beta} \mid \beta \in B\right\}$ we see that $\mathcal{Q}$ is a locally finite refinement of $\mathbb{B}$. Since $\{$ refines $\mathbb{B}$ and $\mathbb{B}$ refines $\mathfrak{B}^{\prime}$ we see that $\mathfrak{U}^{\prime}$ refines $\mathfrak{B}^{\prime}$. It is also clear that $\mathfrak{U}^{\prime}$ is a locally finite open cover of $X$, and that $\mathfrak{U}^{\prime}$ can be indexed on a set $S$ such that $\boldsymbol{x}(S) \leq$ $\boldsymbol{N}(A)\left[\boldsymbol{K}(S)<\boldsymbol{\aleph}_{0}\right]$ when $A$ is infinite [finite]. To complete the proof we show that $\mathfrak{U}^{\prime}$ has the property (11), and, then we modify $\mathfrak{U}^{\prime}$ outside of $\bigcup\left\{F_{j} \mid 1 \leq\right.$ $j \leq n+1\}$ to obtain $\mathfrak{U}$ which has both properties (10) and (11). (The proof 
that $\mathfrak{U}^{\prime}$ satisfies $(11)$ is given below.) Now we modify $\mathfrak{U}^{\prime}$. To do this we make use of Lemma 1: Using (12) and the fact that $\{$ is a pairwise disjoint collection we can show that

$$
\left.H \subset \operatorname{int}\left(\cup\left\{O \mid O \in \S_{j}(\mathfrak{M})^{\prime}\right) ; 1 \leq j \leq n+1\right\}\right)
$$

We choose an open set $D$ such that

$$
H \subset D \subset \operatorname{cl}(D) \subset \operatorname{int}\left(\bigcup\left\{O \mid O \in \bigcap_{j}\left(\mathfrak{U}^{\prime}\right) ; 1 \leq j \leq n+1\right\}\right) .
$$

Let $Y=F \cap(X-D)$ and let $Z=X-H$, then $Y$ is a closed subset of the open set $Z$ and $\operatorname{dim} Y \leq n$. Then using $\mathfrak{U}^{\prime}$ as we have already computed, and indexed on the set $S$, we can apply Lemma 1 to obtain the desired $\mathfrak{U}$. Thus, it only remains to prove the three statements that we alluded to above:

Proof that $\Re$ is a separated collection. To see this we suppose that 0 $\cap F_{j}$ and $P \cap F_{k}$ are distinct members of $\Re$. First, we note that

$$
\left(O \cap F_{j}\right) \cap \operatorname{cl}\left(P \cap F_{k}\right) \subset\left(O \cap F_{j}\right) \cap \operatorname{cl}(P) \cap F_{k} \subset(O \cap \operatorname{cl}(P)) \cap F_{k} \cap F_{j}
$$

If $j=k$, then since $O \cap F_{j}$ and $P \cap F_{k}$ are distinct we see that $O$ and $P$ must be distinct members of $\bigcap_{j}(\mathscr{B})=\bigcap_{k}(\mathscr{B})$. Consequently, these nodes are disjoint and we can use the N. C. P. in this case to show $O \cap \mathrm{cl}(P)=\varnothing$. If $j \neq k$, then

$$
(O \cap \mathrm{cl}(P)) \cap F_{k} \cap F_{j} \subset O \cap \mathrm{cl}(P) \cap H_{r} \quad \text { where } r=\min \{k, j\} .
$$

If $j<k$, then we can use $r=j, O \in \bigcap_{j}(\mathbb{B})$ and (13) to show $O \cap H_{r}=\varnothing$. If $k<j$, then we can use $P \in \bigcap_{k}$ (DSB) and $O \in \bigcap_{j}(\mathbb{D S})$ and the N.C.P. to show that $O \cap \mathrm{cl}(P)=\varnothing$. Thus we have exhausted all cases and we see that $\Re$ is separated.

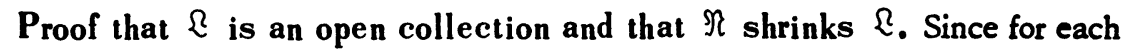
$k>j, O \cap F_{j} \cap F_{k} \subset O \cap H_{j}$, we can apply (13) to see that $\bigcup\left\{F_{k} \mid k>j\right\}$ and $N_{\beta}=O \cap F_{j}$ are disjoint. Also, we can use the N.C.P. to show that $\bigcup\left\{P \mid P \in \bigcap_{k}(\mathbb{R}) ; k<j\right\}$ is a closed set, and, we can use the definition of $\Re_{j}$ to show that this set and $N_{\beta}$ are disjoint.

Proof that $\mathfrak{U}^{\prime}$ satisfies (11). To see that for each $j, 1 \leq j \leq n+1$,

$$
F_{j} \subset X-\cup\left\{0 \mid 0 \in \bigcap_{j}\left(\mathbb{U}^{\prime}\right)\right\} \text {. }
$$

we let $O \in \bigcap_{j}\left(\mathfrak{U}^{\prime}\right)$ and suppose $x \in O \cap F_{j}$. Since $\mathfrak{Z}$ is a pairwise disjoint collection we see that either $x \in P \in S_{j}(\mathbb{B})$ or $x \in P \in S_{j-1}$ (BB) whenever $x \in O \in \bigcap_{j}\left(\mathfrak{U}^{\prime}\right)$. Using this we can conclude that in either case there is an $L_{\beta} \in \Re$ such that $x \in P \cap L_{\beta} \cap F_{j}$. First, if $P \in \bigcap_{j}(\mathbb{S})$, then $x$ is in a $(j+1)$ node of $\mathfrak{U}^{\prime}$. But this is absurd since $x$ is in the $j$-node $O$ of $\mathfrak{U}^{\prime}$. Secondly, 
we suppose that $P \in \bigcap_{k}(\mathbb{B S})$ where $j-1=k<j$. Then, we can use $x \in L_{\beta} \cap P$ and $k<j$ to show that an index $j\left(L_{\beta}\right)<j$ was used in the definition of this particular $L_{\beta}$. However, with this index and the definition of $L_{\beta}$, we can see that $L_{\beta} \cap F_{j}=\varnothing$. Since we have run out of cases we conclude that for each $j, 1 \leq j \leq n+1$, the $j$-nodes of $\mathfrak{U}^{\prime}$ miss $F_{j}$.

Lemma 4. Let $n, X$, and $X, 1 \leq j \leq n+1$, be as in the hypothesis of Theorem 7. Let $\mathbb{B}$ be an open cover of $X$. For each $j, 1 \leq j \leq n+1$, let $\mathbb{B}_{j}$ be a decomposition of $X$ such that $X\left(\mathbb{R}_{j}\right) \leq \tau$ and lord $\mathbb{B}_{j} \leq 2$. Also, let Bdry $\mathbb{B}_{1}, \ldots$, Bdry $\mathbb{B}_{n+1}$ satisfy $P(n-1)$. For each $j$ let

$$
\text { Bdry } \mathbb{B}_{j}=\bigcup\left\{F \mid F \in \mathfrak{F}_{j}\right\}, \quad 火\left(\mathfrak{F}_{j}\right) \leq r,
$$

where $\mathfrak{F}_{j}$ is a discrete closed collection which shrinks an open collection $\mathbb{B}_{j}$. Then for each $j, 1 \leq j \leq n+1$, there are three discrete collections $\mathfrak{U}_{j}(r)$, $1 \leq r \leq 3$, such that

$$
\mathfrak{u}_{j}=\bigcup\left\{\mathfrak{u}_{j}(r) \mid 1 \leq r \leq 3\right\}
$$

is a decomposition of $X, \boldsymbol{x}\left(\mathfrak{U}_{j}\right) \leq \tau$, and $\mathfrak{U}_{j}(r) \cap \mathfrak{U}_{j}(s)=\varnothing$ whenever $r \neq s$. In addition, the following properties hold:

(14) for each $j, 1 \leq j \leq n+1$, lord $\mathfrak{U}_{j} \leq 2$;

(15) for each $j, 1 \leq j \leq n+1,\left\{\mathrm{cl}(U) \mid U \in \mathfrak{U}_{j}(1)\right\}$ refines $\mathfrak{B}$ and $\bigcup\left\{\mathfrak{u}_{j}(1) \mid\right.$ $1 \leq j \leq n+1\}$ covers $X$;

(16) if $x \in \mathrm{Bdry} \mathfrak{U}_{j}$ for some $j \in\{1, \ldots, n+1\}$, then there exist distinct members $U_{1}, U_{2} \in \mathfrak{U}_{j}$ such that $x \in B\left(U_{1}\right) \cap B\left(U_{2}\right)$;

(17) for each $j, 1 \leq j \leq n+1, \mathfrak{u}_{j}$ covers $X_{j}$;

(18) for each $j, 1 \leq j \leq n+1$, Bdry $\mathfrak{U}_{j} \cap$ Bdry $\mathbb{B}_{j}=\varnothing$;

(19) for each $j, 1 \leq j \leq n+1, \mathfrak{U}_{j}(1) \cup U_{j}$ (3) refines $\mathfrak{B}_{j}$;

(20) for each $j, 1 \leq j \leq n+1, \mathfrak{U}_{j}(1) \cup \mathfrak{U}_{j}(2)$ is a discrete collection; and

(21) for each $j, 1 \leq j \leq n+1, \mathfrak{U}_{j}(2)$ puffs-up $\mathfrak{F}_{j}$ in $\mathbb{B}_{j}$ and $\mathrm{cl}\left[\mathfrak{U}_{j}(2)\right]$ shrinks ${ }_{j}$.

Proof. First, we mention that property (16) is sort of an add-on property. By this we mean that if $\mathfrak{U}_{j}^{\prime}, 1 \leq j \leq n+1$, were constructed having all corresponding properties except (16), then we could modify each $\mathfrak{U}_{j}^{\prime}$ to obtain the desired $\mathfrak{U}_{j}$. More precisely, let $j \in\{1, \ldots, n+1\}$ and $r \in\{1,2,3\}$ be fixed. Then $U \in \mathfrak{U}_{j}(r)$ whenever there is a $U^{\prime} \in \mathfrak{U}_{j}^{\prime}(r)$ such that

$$
U=X-\bigcup\left\{\mathrm{cl}(V) \mid V \in \mathfrak{U}_{j}^{\prime} ; \quad V \neq U^{\prime}\right\} \text {. }
$$

If $U$ and $U^{\prime}$ correspond in this manner, then we can show that $\operatorname{cl}(U)=\operatorname{cl}\left(U^{\prime}\right)$, $U^{\prime} \subset U$, and $B(U) \subset B\left(U^{\prime}\right)$. With this and the properties of. $\mathfrak{u}_{j}^{\prime}, 1 \leq j \leq n+1$, 
we can show that the decompositions

$$
\mathfrak{u}_{j}=\mathfrak{U}_{j}(1) \cup \mathfrak{u}_{j}(2) \cup \mathfrak{u}_{j}(3), \quad 1 \leq j \leq n+1,
$$

have all the required properties. Thus, we can ignore (16) in the following construction.

To begin this construction, we set up an application of Lemma 3. For this, we let $F=X$, Bdry $B_{j}=F_{j}$ for $1 \leq j \leq n+1$, and $B^{\prime}$ be a locally finite open cover of $X$ indexed on $A$ such that $\left\{c l(V) \mid V \in B^{\prime}\right\}$ refines $B$. Consequently, we obtain the resultant $\mathfrak{U}, \boldsymbol{\kappa}(\mathfrak{U}) \leq \boldsymbol{\aleph}(A)$, which satisfies $(10)$ and (11). We can use the se properties and the fact that $F=X$ to apply Lemma 2. We place primes on these new discrete collections, i.e., we obtain $\mathfrak{u}_{j}^{\prime}(1), 1$ $\leq j \leq n+1$. We now let $j \in\{1, \ldots, n+1\}$ and define

$$
\mathfrak{U}_{j}(1)=\left\{U \cap W \mid U \in \mathfrak{U}_{j}^{\prime}(1) ; W \in \mathfrak{M}_{j}\right\}
$$

(Note $\left.\mathcal{K}\left(\mathfrak{U}_{j}(1)\right) \leq \mathcal{N}(A)_{0}\right)$ The properties of $\mathfrak{U}_{j}^{\prime}, 1 \leq j \leq n+1$, show that each $\mathfrak{U}_{j}(1)$ is discrete and Bdry $\mathfrak{U}_{j}(1) \cap X_{j}=\varnothing$. Also, since $\bigcup_{\mathrm{cl}}\left[\mathfrak{U}_{j}(1)\right] \cap$ $\left(\bigcup\left\{F \mid F \in \mathfrak{F}_{j}\right\}\right)=\varnothing$ for each $j$, and, since each $\mathfrak{F}_{j}$ is a discrete closed collection, we can puff-up $\left\{F \mid F \in \mathfrak{F}_{j}\right\}$ in $\mathbb{F}_{j}$ to a discrete open collection $\mathfrak{U}_{j}$ (2) such that $\mathrm{cl}\left[\mathfrak{U}_{j}(2)\right]$ shrinks $\otimes_{j}$ and $\left(\bigcup_{\mathrm{cl}}\left[\mathfrak{U}_{j}(1)\right]\right) \cap\left(\bigcup_{\mathrm{cl}}\left[\mathfrak{U}_{j}(2)\right]\right)=\varnothing$. Further, since $X_{j}$ is zerodimensional we can assume that $U \in \mathfrak{U}_{j}(2)$ implies that $B(U) \cap X_{j}=\varnothing$. (Note $\boldsymbol{\alpha}\left(\mathfrak{U}_{j}(2)\right) \leq \boldsymbol{N}(A)$.) For each $j, 1 \leq j \leq n+1$, we let $R_{j}=U_{c l}\left[\mathfrak{u} \mathfrak{u}_{j}(1)\right]$ and $S_{j}=\bigcup_{\operatorname{cl}}\left[\overline{\mathfrak{U}}_{j}(2)\right]$. For each $j, 1 \leq j \leq n+1$, we let

$$
\mathfrak{U}_{j}(3)=\left\{\left[X-\left(R_{j} \cup S_{j}\right)\right] \cap W \mid W \in \mathbb{B}_{j}\right\}_{\bullet}
$$

(Note $\boldsymbol{K}\left(\mathfrak{u}_{j}(3)\right) \leq \boldsymbol{N}(A)$.) All the properties, except (16), now clearly follow from the constructions and the remarks above. From the statements at the beginning of the proof we conclude that we are finished.

Proof of the decomposition theorem (Theorem 7). The proof is by induction on the index $i$. For $i=1$ we can apply Lemma 2: Let $\mathfrak{U}$ be a locally finite open cover of $X$ such that mesh $\mathfrak{U}<1, \mathcal{N}(\mathfrak{U}) \leq \mathcal{X}(A)$, and ord $\mathfrak{U} \leq n+1$. Let $F_{1}=\cdots=F_{n+1}=\varnothing$. Thus, we obtain $\mathfrak{U}_{j}(1), 1 \leq j \leq n+1$. We let

$$
\mathfrak{B}_{1 j}=\mathfrak{U}_{j}(1) \cup\left\{X-\left(\cup \operatorname{cl}\left[\mathfrak{U}_{j}(1)\right]\right)\right\}
$$

for $1 \leq j \leq n+1$. The properties $(5)_{1}, \ldots,(9)_{1}$ are vacuously satisfied. Properties $(1)_{1},(2)_{1}$, and $(4)_{1}$ follow easily from the properties of $\mathfrak{U}_{j}(1)$, $1 \leq j \leq n+1$. Property (3) 1 can be obtained if we modify each $\mathbb{B}_{1 j}$ in the same way that $\mathfrak{U}_{j}^{\prime}$ was modified to obtain $\mathfrak{U}_{j}$ in the proof of Lemma 4 . 
Moreover, since the modification does not affect the truth of $(1)_{1},(2)_{1},(4)_{1}$, $(5)_{1}, \ldots,(9)_{1}$ we conclude that the collections $\mathfrak{B}_{1 j}, 1 \leq j \leq n+1$, have been constructed. (Note $\mathcal{N (}\left(\mathbb{R}_{1 j}\right) \leq \boldsymbol{\aleph}(A)$ since $A$ is infinite.)

Now suppose that $B_{k j}, 1 \leq j \leq n+1$ and $k \leq i-1$, have been constructed. We will construct $\mathfrak{B}_{i j}, 1 \leq j \leq n+1$, with properties $(1)_{i}, \ldots,(9)_{i}$. To do this we will make an application of Lemma 4 . First, we let $\mathfrak{B}$ be an open cover of $X$ of mesh less than $(1 / i)$. Then we define

$$
\mathbb{B}_{j}=\left\{W_{1} \cap \ldots \cap W_{i-1} \mid W_{k} \in \mathbb{B}_{k j} ; 1 \leq k \leq i-1\right\} \text { for } 1 \leq j \leq n+1 \text {. }
$$

We can see that each $\mathbb{B}_{j}$ is a decomposition of $X$ such that $X\left(\mathbb{B}_{j}\right) \leq \tau$, lord $\mathbb{B}_{j} \leq 2$, and

$$
\operatorname{Bdry} \mathbb{B}_{j}=\bigcup\left\{\text { Bdry } \mathbb{B}_{k j} \mid 1 \leq k \leq i-1\right\} \text {. }
$$

Thus, using $(4)_{k}, 1 \leq k \leq i-1$, we can show that Bdry $\mathbb{B}_{1}, \ldots$, Bdry $\mathbb{B}_{n+1}$ satisfy $P(n-1)$. For $1 \leq j \leq n+1$ we let

$$
\xi_{j}=\left\{B\left(W_{1}\right) \cap B\left(W_{2}\right) \mid W_{1} \neq W_{2} ; W_{1}, W_{2} \in \mathbb{R}_{k j} \text { for some } k \leq i-1\right\} \text {. }
$$

We can use $(3)_{k}, 1 \leq k \leq i-1$, and $(*)$ to show $B d r y \mathbb{B}_{j}=\bigcup\left\{F \mid F \in \mathfrak{F}_{j}\right\}$. For each $k, 1 \leq k \leq i-1$, we can use $(1)_{k}$ to show that

$$
\left\{B\left(W_{1}\right) \cap B\left(W_{2}\right) \mid W_{1} \neq W_{2} ; W_{1}, W_{2} \in \mathbb{B}_{k j}\right\}
$$

is a discrete collection. Then we can use $(5)_{k}, 1 \leq k \leq i-1$, to show that for each $j, 1 \leq j \leq n+1, \mathfrak{F}_{j}$ is a discrete collection. (Note $\boldsymbol{\alpha}\left(\mathfrak{F}_{j}\right) \leq \pi_{0}$ ) Now we can define the collections $B_{j}, 1 \leq j \leq n+1$ : Let $k$ be a member of $\{1, \ldots, i-1\}$. Let $F \in \mathfrak{F}_{j}$ be fixed, where $F=B\left(W_{1}\right) \cap B\left(W_{2}\right)$ with $W_{1}, W_{2}$ $\in B_{k j}$ Using $(6)_{i-1}$ if $k=i-1$, or $(7)_{i-1}$ and $(9)_{i-1}$ if $k<i-1$, we can show that there is a unique collection of open sets

$$
\left\{W_{r} \mid 1 \leq r \leq i-1 ; r \neq k ; W_{r} \in \mathbb{R}_{r j}\right\}
$$

whose intersection contains $F$. Also, since lord $\mathbb{B}_{k j} \leq 2$ there is an open set $H$ such that $F \subset H$ and $H$ meets at most two members of $\mathbb{R}_{k j^{\bullet}}$ We let

$$
G(F)=H \cap G_{i}(F) \cap\left(\cap\left\{W_{r} \mid 1 \leq r \leq i-1 ; r \neq k ; W_{r} \in \mathbb{B}_{r j}\right\}\right) .
$$

Now for each $j, 1 \leq j \leq n+1$, we define $\mathbb{B}_{j}=\left\{G(F) \mid F \in \mathfrak{F}_{j}\right\}$. Clearly each $\mathfrak{F}_{j}$ shrinks $B_{j}$. Now we can apply Lemma 4 and, consequently, we can define $\mathbb{B}_{i j}=U_{j}, 1 \leq j \leq n+1$. Using $(14), \ldots$, (21) we can easily show that $\mathbb{B}_{i j}$, 
$1 \leq j \leq n+1$, satisfies all the requirements for the $i$ th collections, e.g., (15) and (19) imply $(2)_{i}$. Since this completes the induction step we see that the proof is finished. $\square$

7. The maps $h$ and $\theta$. Throughout this section we let $X$ be an $n$-dimensional metric space, $n \geq 0$, of infinite weight $r=\aleph(A)$. We suppose that

$$
A=\bigcup\left\{A_{i j} \mid i \geq 1 ; 1 \leq j \leq n+1\right\}
$$

is a partition of $A$ into sets $A_{i j}$ such that for all $i$ and all $j, \boldsymbol{\aleph}(A)=\boldsymbol{\aleph}\left(A_{i j}\right)$. Then for our particular $X$ we can choose zero-dimensional subspaces $X_{j}$, $1 \leq j \leq n+1$, and apply Theorem 7. Consequently, we obtain the decompositions $\mathbb{B}_{i j}=\left\{W\left(a_{i j}\right) \mid \alpha_{i j} \in A_{i j}\right\}$.

Let $a \in N(A)^{n+1}$. Then $a$ consists of $n+1$ sequences $a^{j}=\left\{\alpha_{i j} \mid i \geq\right.$ $1\}, 1 \leq j \leq n+1$, where $a^{j}$ is the $j$ th component of $\alpha$. We will denote $\alpha$ by $\left\{a_{i j}\right\}$ where it is understood that $i \geq 1$ and $1 \leq j \leq n+1$.

With this notation and the decompositions we can define $T \subset N(A)^{n+1}$ and $h: T \rightarrow X$ as follows: $a=\left\{a_{i j}\right\} \in T$ if, and only if,

$$
h(\alpha)=\bigcap\left\{\mathrm{cl}\left(W\left(\alpha_{i j}\right)\right) \mid i \geq 1 ; 1 \leq j \leq n+1\right\} \neq \varnothing .
$$

Since each $\mathbb{B}_{i j}$ is a decomposition of $X$ we can use $(2)_{i}, i \geq 1$, to show that $h$ is well defined.

Theorem 8. The map $h: T \rightarrow X$ is a continuous quotient map.

Proof. First we show that $h$ is continuous: Let $a=\left\{a_{k j}\right\} \in T$. Suppose we say that $\beta=\left\{\beta_{k j}\right\} \in T$ is $i$-close to $\alpha$ if there is an $i$ such that for each $j, 1 \leq j \leq n+1, \alpha_{k j}=\beta_{k j}$ for $1 \leq k \leq i$. From (2) $i$ we can easily show that the distance between $h(\alpha)$ and $h(\beta)$ is less than $1 / i$ whenever $\alpha$ and $\beta$ are $i$-close. Thus, the continuity of $h$ is obvious.

We now show that $h$ is quotient: To see this we suppose $h^{-1}(Q)$ is open. It suffices to show that $Q$ is open. Let $x \in Q$. We construct a neighborhood of $x$ which is contained in $Q$. Let $a \in h^{-1}(x)$. Then we denote the basic open set in $N(A)^{n+1}$ of the form

$$
\left\langle\alpha_{11}, \ldots, \alpha_{i 1}\right\rangle \prod \ldots \prod\left\langle\alpha_{1 n+1}, \ldots, \alpha_{i n+1}\right\rangle
$$

by $\left\langle a_{i j}\right\rangle$. We can choose $i$ large enough so that if there is a $j, 1 \leq j \leq n+$ 1 , such that $x \in \mathrm{Bdry} \mathbb{B}_{k j}$, then $k<i$. Further, since $h^{-1}(x)$ is a finite set and $b^{-1}(Q)$ is open we can assume that $i$ is large enough so that U $\left\{\left\langle a_{i j}\right\rangle \mid \alpha \in h^{-1}(x)\right\} \subset h^{-1}(Q)$. We let 


$$
\begin{aligned}
V= & {\left[\cap\left\{W \mid x \in W \in \mathbb{B}_{k j} ; 1 \leq k \leq i \text { and } 1 \leq j \leq n+1\right\}\right] } \\
& \cap\left[\bigcup\left\{c l(W) \mid x \in B(W) ; W \in \mathbb{N}_{k j} ; 1 \leq k<i \text { and } 1 \leq j \leq n+1\right\}\right] .
\end{aligned}
$$

Then we can use the properties stated in Theorem 7 , together with the fact that $y \in V$ and $h(\beta)=y$ implies $\beta \in \bigcup\left\{\left\langle\alpha_{i j}\right\rangle \mid \alpha \in h^{-1}(x)\right\}$, to show that $V$ is a neighborhood of $x$ which is contained in $Q$.

We use these same decompositions to define the map $\theta$. First, however, we will need the concepts of last ancestor and no ancestor: Let $j \in\{1, \ldots$, $n+1\}$ be fixed. Let $\alpha_{k j} \in A_{k j}$ for $k=1, \ldots, i$, be such that $\bigcap\left\{\mathrm{cl}\left(W\left(\alpha_{k j}\right)\right) \mid k=1, \ldots, i\right\} \neq \varnothing$. If $W\left(\alpha_{i j}\right) \cap$ Bdry $\mathbb{2}_{k j}=\varnothing$ for each $k<i$, then $\alpha_{i j}$ is said to have no ancestor. Otherwise, by properties (3) $)_{k}, k<i$, and $(7)_{i}$ there is a unique $r<i$ and a unique $\beta_{r j} \in A_{r j}$ such that $\beta_{r j} \neq a_{r j}$ and $W\left(\alpha_{i j}\right) \cap B\left(W\left(\alpha_{r j}\right)\right) \cap B\left(W\left(\beta_{r j}\right)\right) \neq \varnothing$; this $\beta_{r j}$ is called the 1st-ancestor of $\alpha_{i j}$ with respect to $\alpha_{1 j}, \ldots, \alpha_{(i-1) j}$. The 2 nd-ancestor of $\alpha_{i j}$ with respect to $\alpha_{1 j}, \ldots, \alpha_{(i-1) j}$ is defined to be the 1st-ancestor of $\beta_{r j}$ with respect to $\alpha_{1 j}, \ldots, \alpha_{(r-1) j}$. The process of determining ancestors of $\alpha_{i j}$ terminates after at most $i-1$ steps, and the final ancestor determined is called the last ancestor of $\alpha_{i j}$ with respect to $\alpha_{1 j}, \ldots, \alpha_{(i-1) j}$.

Now we define the map $\theta$ : Let $\alpha=\left\{\alpha_{i j}\right\} \in T$. For each $i \geq 1$, and each $j, 1 \leq j \leq n+1$, let $\gamma_{i j}=a_{i j}$ if $\alpha_{i j}$ has no ancestor, otherwise, let $\gamma_{i j}$ be the last ancestor of $\alpha_{i j}$ with respect to $\alpha_{1 j}, \ldots, \alpha_{(i-1) j}$. We call this correspondence $\theta: T \rightarrow N(A)^{n+1}$, i.e., $\theta(a)=\theta\left(\left\{a_{i j}\right\}\right)=\left\{\gamma_{i j}\right\}=\gamma \in N(A)^{n+1}$. It is easy to see that $\theta$ is well defined and continuous.

Lemma 5. For each $x=h(a) \in X$ there is an $s \in\{1, \ldots, n+1\}$ and an infinite number of $i \in\{1,2, \ldots\}$ such that $\theta(\alpha)_{i s}=\alpha_{i s}, h(\alpha) \in W\left(\alpha_{i s}\right)$, and $\operatorname{dia}\left(W\left(\alpha_{i s}\right)\right)<1 / i$.

Proof. It is clear that for each $i \in\{1,2, \ldots\}$ there is a $j \in\{1, \ldots, n+1\}$ such that property $(2)_{i}$ holds for $x=h(\alpha)$. Consequently, for $x=h(\alpha)$, there is at least one $j$, say $j=s$, for which property $(2)_{i}$ holds for an infinite number of $i$. By definition of no ancestor it follows that for these $i, \theta(\alpha)_{i s}=$ $a_{i s}$ The other properties clearly follow.

Lemma 6. Let $\alpha, \beta \in T$ with $\alpha_{r s} \neq \beta_{r s}$ where $r \in\{1,2, \ldots\}$ and $s \epsilon$ $\{1, \ldots, n+1\}$. Suppose $i=r+1$ and $h(\alpha), h(\beta) \in W \in \mathbb{B}$ is and $\varnothing \neq$ $B\left(W\left(\alpha_{r s}\right)\right) \cap B\left(W\left(\beta_{r s}\right)\right) \subset W$. Then $\theta(\alpha)_{i s} \neq \theta(\beta)_{i s}$.

Proof. Since $h(\alpha), h(\beta) \in W \in \mathbb{B}_{i s}$ it follows that $\alpha_{i s}=\beta_{i s}$, and since $\varnothing \neq B\left(W\left(\alpha_{r s}\right)\right) \cap B\left(W\left(\beta_{r s}\right)\right) \subset W$, for $\alpha_{r s} \neq \beta_{r s}$, it follows that $\beta_{r s}$ is the 1st-ancestor of $\alpha_{i s}$ (relative $\alpha_{1 s}, \ldots, \alpha_{r s}$ ) and that $\alpha_{r s}$ is the 1st-ancestor 
of $\beta_{\text {is }}$ (relative $\beta_{1 s}, \ldots, \beta_{r s}$ ). Property (6), shows that either $\alpha_{r s}$ or $\beta_{r s}$ has no ancestor. It follows, by definition of $\theta$, that $\theta(\alpha)_{i s} \neq \theta(\beta)_{i s}$.

Theorem 9. The map $\theta: T \rightarrow \theta(T) \subset N(A)^{n+1}$ is a homeomorphism.

Proof. We only need to show that $\theta$ is one-one and that $\theta^{-1}$ is continuous. First, we show that $\theta$ is one-one: Let $\alpha, \beta \in T$ and assume that $\alpha_{r s} \neq \beta_{r s}$ for some $r$ and some $s$. Suppose that $h(\alpha)=h(\beta)$. Then

$$
h(\alpha)=h(\beta) \in B\left(W\left(\alpha_{r s}\right)\right) \cap B\left(W\left(\beta_{r s}\right)\right) \neq \varnothing
$$

and we can use $(8)_{i}$ for $i=r+1$ to see that there is a $W \in \mathbb{P}_{i s}$ such that $B\left(W\left(\alpha_{r s}\right)\right) \cap B\left(W\left(\beta_{r s}\right)\right) \subset W$. By Lemma 6 we see that $\theta(\alpha)_{i s} \neq \theta(\beta)_{i s}$. We can now suppose that $h(\alpha) \neq h(\beta)$. Then by Lemma 5 there is an $s \epsilon$ $\{1, \ldots, n+1\}$ and an infinite number of $i \in\{1,2, \ldots\}$ such that $\theta(\alpha)_{i s}=$ $\alpha_{i s}, h(\alpha) \in W\left(\alpha_{i s}\right)$, and $\operatorname{dia}\left(W\left(\alpha_{i s}\right)\right)<1 / i$. Since $h(\alpha) \neq h(\beta)$ we know that these points are some distance $e$ apart. If we take $i$ such that $(1 / i)<$ $(e / 2), h(\alpha) \in W\left(\alpha_{i s}\right), \theta(\alpha)_{i s}=\alpha_{i s}$, and $\operatorname{dia}\left(W\left(\alpha_{i s}\right)\right)<1 / i$, then $h(\beta) \notin$ $\mathrm{cl}\left(W\left(\alpha_{i s}\right)\right)$ and, consequently, $\theta(\alpha)_{i s} \neq \theta(\beta)_{i s}$.

Secondly, we show that $\theta^{-1}$ is continuous: Let $a \in T$ and $m \in\{1,2$,

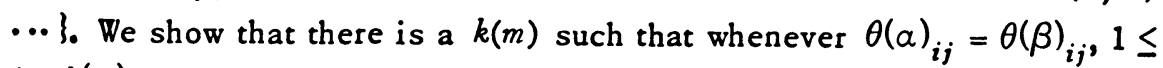
$i \leq k(m)$ and $1 \leq j \leq n+1$, then $\alpha_{i j}=\beta_{i j}$ for $1 \leq i \leq m$ and $1 \leq j \leq n+1$. We assume, without loss of generality, that if $h(\alpha) \in B\left(W\left(\alpha_{r s}\right)\right)$ for some $r$ and some $s$, then $r<m$. Now consider

$$
G=\bigcap\left\{W\left(a_{i j}\right) \mid 1 \leq i \leq m, 1 \leq j \leq n+1, \text { and } h(\alpha) \in W\left(a_{i j}\right)\right\} \text {. }
$$

Then $G$ is open and $h(\alpha) \in G$ implies, if we use Lemma 5 , that there is an $s \in\{1, \ldots, n+1\}$ and $a(m)=r>m$ such that $\theta(\alpha)_{r s}=\alpha_{r s}$ and $h(\alpha) \epsilon$ $W\left(\alpha_{r s}\right) \subset \mathrm{cl}\left(W\left(\alpha_{r s}\right)\right) \subset G$. If $\theta(\alpha)_{r s}=\theta(\beta)_{r s}=\alpha_{r s}$, then $\beta_{r s}=\alpha_{r s}$ and we see that $h(\beta) \in \mathrm{cl}\left(w\left(\alpha_{r s}\right)\right) \subset G$. Consequently, $\beta_{i j}=\alpha_{i j}$ for $1 \leq i \leq m$ and $1 \leq j \leq n+1$, except possibly for those pairs in $\left\{(i, j) \mid h(\alpha) \in B\left(W\left(\alpha_{i j}\right)\right)\right\}$. However, if we assume that $\alpha_{u v} \neq \beta_{u v}$ for $(u, v)$ in this set, then we could contradict $\theta(\alpha)_{i v}=\theta(\beta)_{i v}$ for $i=u+1 \leq m<k(m)$. (Indeed, suppose $\alpha_{u v} \neq \beta_{u v}$. Now $i>u$ and $(5)_{i}$ show $h(\alpha) \in W\left(\alpha_{i v}\right) \in \mathbb{R}_{i v}$. Then $i \leq m, h(\alpha) \in W\left(\alpha_{i v}\right)$ and $h(\beta) \in G$ show $h(\beta) \in W\left(\alpha_{i v}\right)$. Thus $h(\alpha) \in B\left(W\left(\alpha_{u v}\right)\right),(3)_{u}$ and $(7)_{i}$ can be used to show

$$
B\left(W\left(\alpha_{u \nu}\right)\right) \cap B\left(W\left(\beta_{u v}\right)\right) \neq \varnothing .
$$

Further, $(9)_{i}$ shows 


$$
\varnothing \neq B\left(W\left(\alpha_{u v}\right)\right) \cap B\left(W\left(\beta_{u v}\right)\right) \subset W\left(\alpha_{i v}\right) \in \mathbb{B}_{i v},
$$

and an obvious application of Lemma 6 would then show that $\theta(\alpha)_{i v} \neq \theta(\beta)_{i v}$.) Thus we see that $\theta^{-1}$ is continuous.

We have defined $R$ to be an equivalence relation in $N(A)$. But now however, we are concerned with $N(A)^{n+1}$, and we will let for $\gamma, \delta \in N(A)^{n+1}$, $\gamma R \delta$ mean that for every $j, 1 \leq j \leq n+1$, the $j$ th components of $\gamma$ and $\delta$ are $R$-related.

Lemma 7. Let $R, \theta$, and $T$ be defined as above. Let $x=h(\alpha)$. Then $\theta(\alpha) R \gamma$ implies there is a $\beta \in h^{-1}(x)$ such that $\theta(\beta)=\gamma$.

Proof. First, suppose that we can prove the statement for the case where $\theta(\alpha)$ and $\gamma$ differ at exactly one of the $j$ th components, $j \in\{1, \ldots, n+1\}$. Then for the case where $\theta(\alpha)$ and $\gamma$ differ at exactly two of the $j$ th components, we could obtain a $\delta \in N(A)^{n+1}, \delta R \theta(a)$ and $\delta R \gamma$, that differs from $\theta(a)$ at one of these components and that differs from $\gamma$ at the other, while agreeing with $\theta(\alpha)$ and $\gamma$ on the remaining components. We can then apply the first case twice to obtain the desired $\beta \in T$ such that $\theta(\beta)=\gamma_{\bullet}$

The general case follows by a similar induction argument. Hence, we assume that $\theta(\alpha)$ and $\gamma$ differ at exactly one component $j \in\{1, \ldots, n+1\}$. To prove this case we will show below that there is a unique $r \in\{1,2, \cdots\}$ and $a$ unique $\epsilon_{r j} \in A_{r j}$ such that $\epsilon_{r j} \neq \alpha_{r j}$, and

$$
B\left(W\left(\alpha_{r j}\right)\right) \cap B\left(W\left(\epsilon_{r j}\right)\right)=\bigcap\left\{c l\left(W\left(\alpha_{i j}\right)\right) \mid i \geq 1\right\} .
$$

From this it follows that $h(\alpha) \in \operatorname{Bdry} \mathbb{B}_{r j}$. Then we can define $\beta$ : If $i \epsilon$ $(\{1,2, \ldots\}-\{r\})$ or $s \in(\{1,2, \ldots\}-\{j\})$ we let $\beta_{i s}=\alpha_{i s}$, and, for $i=r$ and $s=j$ we let $\beta_{i s}=\beta_{r j}=\epsilon_{r j}$. From (22) and the definitions of $T$ and $h$ we can see that $\beta \in T$ and that $h(\beta)=h(\alpha)$.

With this result, we can use an argument which has the flavor of the one used in the proof of Lemma 6 to show that the $j$ th components of $\theta(\alpha)$ and $\theta(\beta)$ are unequal but $R$-related. It will then follow that $\theta(\beta)=\gamma$. With these remarks it will thus suffice to show that (22) is true for a unique $r$ and a unique $\epsilon_{r j}$. To see this we let $k$ be an arbitrary integer greater than the tail index of the $j$ th component of $\theta(\alpha)$, and, we let $\epsilon_{r j}$ be the unique 1st-ancestor of $a_{k j}$ with respect to $a_{1 j}, \ldots, \alpha_{(k-1) j} \bullet$ (Now $a_{k j}$ has a 1st-ancestor: Suppose otherwise. Then $\theta(\alpha)_{k j}=a_{k j}$ and it would then follow, since the tail of $\left\{\theta(a)_{i j} \mid i \geq 1\right\}$ is constant and $k$ is greater than the tail index of $\left\{\theta(\alpha)_{i j} \mid i \geq 1\right\}$, that $\alpha_{k j}$ is the last ancestor of $\alpha_{i j}$ whenever $i>k$. But from the definition of an ancestor of $\alpha_{i j}$ relative to $\alpha_{1 j}, \ldots, a_{(i-1) j}$ we can 
infer that any ancestor of $\alpha_{i j}$ is not a member of $\left.\left\{\alpha_{1}, \ldots, \alpha_{(i-1) j}\right\}_{0}\right)$ Thus $r<k$, and we can use this and $(8)_{k}$ to show

$$
B\left(W\left(a_{r j}\right)\right) \cap B\left(W\left(\epsilon_{r j}\right)\right) \subset W\left(a_{k j}\right) .
$$

Now let $i>k$ be arbitrary but fixed. As argued above we know that $\alpha_{i j}$ has a 1st-ancestor $\epsilon_{m j}$ with respect to $\alpha_{1 j}, \ldots, \alpha_{(i-1) j}$. We can show that $m<k$. (If $m \geq k$, then, as argued above, $\alpha_{m j}$ has a 1st-ancestor relative $\left(\alpha_{1 j}, \ldots, \alpha_{(m-1) j}\right)$. By the definition of $\epsilon_{m j}$ we can see that

$$
B\left(W\left(\alpha_{m j}\right)\right) \cap B\left(W\left(\epsilon_{m j}\right)\right) \cap W\left(\alpha_{i j}\right) \neq \varnothing .
$$

With these two statements we can use $(6)_{m}$ to show that $\epsilon_{m j}$ has no ancestor. Thus $\theta(\alpha)_{i j}$ would equal $\epsilon_{m j}$ if $m \geq k$. But this would contradict $\theta(\alpha)_{i j}=$ $\theta(\alpha)_{k j} \in A_{u j}$ for $\left.u \leq r<k_{0}\right)$ Now, since $m<k$ and

$$
B\left(W\left(\alpha_{m j}\right)\right) \cap B\left(W\left(\epsilon_{m j}\right)\right) \subset W\left(\alpha_{i j}\right)
$$

we can use $(7)_{i}$ to show

$$
W\left(\alpha_{i j}\right) \subset W\left(\alpha_{k j}\right)
$$

But then (23), (24), and (25) show that $W\left(a_{k j}\right)$ contains both $B\left(W\left(a_{m j}\right)\right) \cap$ $B\left(W\left(\epsilon_{m j}\right)\right)$ and $B\left(W\left(a_{r j}\right)\right) \cap B\left(W\left(\epsilon_{r j}\right)\right)$. Thus by $(7)_{k}$ we see that $m=r$. Therefore, since $i$ was arbitrary we have

$$
B\left(W\left(a_{r j}\right)\right) \cap B\left(W\left(\epsilon_{r j}\right)\right) \subset W\left(a_{i j}\right) \text { for all } i \geq k \text {. }
$$

It follows from $(8)_{i}$ that $(22)$ is true.

Using the results above we can show that $\theta$ has the following property: For each $x \in X$ there is a $z \in J(r)^{n+1}, z$ has at most $n$ rational coordinates, such that $\theta$ maps $h^{-1}(x)$ onto $\left(p^{n+1}\right)^{-1}(z)$. To show this we let $x \in X$ and define $z=p^{n+1}\left(\theta\left(h^{-1}(x)\right)\right.$ ). (Clearly $z$ is unique: Suppose $h(\alpha)=h(\beta)=x$. If $\theta(\alpha)^{j} \neq \theta(\beta)^{j}$, let $k=\min \left\{i \mid \alpha_{i j} \neq \beta_{i j}\right\}_{0}$ Then $x \in B\left(W\left(\alpha_{k j}\right)\right) \cap B\left(W\left(\beta_{k j}\right)\right)$ and using an argument similar to the one in the proof of Lemma 6 we can show that $\theta(\alpha)^{j}$ and $\theta(\beta)^{j}$ are properly related.) Then Lemma 5 and the fact that $A_{i s} \cap A_{k s}=\varnothing$ for $i \neq k$ show that there is an $s \in\{1, \ldots, n+1\}$ such that the s-component of each element in $\theta\left(b^{-1}(x)\right)$ does not have a constant tail. We can easily see that it follows that the $s$-component of $p^{n+1}\left(\theta\left(b^{-1}(x)\right)\right)=z$ is irrational. The onto part of the property follows directly from Lemma 7.

\section{BIBLIOGR A PHY}

1. S. Lipscomb, Imbedding one-dimensional metric spaces, Dissertation, University of Virginia, Charlottesville, Va., 1973. 
2. S. Lipscomb, A universal one-dimensional metric space, TOPO 72- General Topology and Its Applications, Second Pittsburgh Internat. Conf., Lecture Notes in Math., vol. 378, Springer-Verlag, New York, 1974.

3. J. Nagata, A survey of dimension theory, General Topology and Its Relations to Modern Analysis and Algebra II, (Proc. Second Prague Sympos., 1966), Academia, Prague, 1967.

4. - A remark on general imbedding theorems in dimension theory, Proc. Japan Acad. 39 (1963), 197-199. MR 29 \#1616.

5. - Modern dimension theory, Bibliotheca Math., vol. 6, Interscience, New York, 1965. MR 34 \#8380.

6. G. Nöbeling, Über eine $n$-dimensionale Universalmenge im $R_{2 n+1}$, Math. Ann. 104 (1930), 71-80.

7. P. Ostrand, Covering dimension in general spaces, General Topology and Appl. 1 (1971), no. 3, 209-221. MR 44 \#5937.

NAVAL WEAPONS LABORATORY, DAHLGREN, VIRGINIA 22448 